\title{
Cannabinoid use and effects in patients with epidermolysis bullosa: an international cross-sectional survey study
}

\author{
Nicholas H. B. Schräder ${ }^{{ }^{*}+}$ (D), Emily S. Gorell ${ }^{2,3{ }^{\dagger}}$, Roy E. Stewart ${ }^{4}$, José C. Duipmans ${ }^{1}$, Nicole Harris ${ }^{3}$, \\ Victoria A. Perez ${ }^{5}$, Jean Y. Tang ${ }^{3}$, André P. Wolff ${ }^{6}$ and Marieke C. Bolling
}

\begin{abstract}
Background: Epidermolysis bullosa (EB) patient anecdotes and case reports indicate that cannabinoid-based medicines (CBMs) may alleviate pain and pruritus and improve wound healing. CBM use has not been characterized in the EB patient population.
\end{abstract}

Objectives: To evaluate CBM use among EB patients, including CBM types, effects on symptoms (e.g., pain and pruritus), disease process (e.g., blistering, wounds, and inflammation), well-being (e.g., sleep, appetite) and concomitant medications.

Methods: English-speaking EB patients or caregivers completed an online international, anonymous, cross-sectional survey regarding CBM use. Respondents reported the types of CBMs, subsequent effects including perceived EB symptom alteration, changes in medication use, and side effects.

Results: Seventy-one EB patients from five continents reported using or having used CBMs to treat their EB. Missing question responses ranged between $0(0 \%)$ and $33(46 \%)$. Most used more than one CBM preparation (mean: $2.4 \pm 1.5$ ) and route of administration (mean: $2.1 \pm 1.1$ ). Topical and ingested were the most common routes. Pain and pruritus were reported retrospectively to decrease by 3 points (scale: $0-10 ; p<0.001$ for both) after CBM use. Most reported that CBM use improved their overall EB symptoms (95\%), pain (94\%), pruritus (91\%) and wound healing (81\%). Most participants (79\%) reported decreased use of pain medications. The most common side-effect was dry mouth (44\%).

Conclusions: CBMs improve the perception of pain, pruritus, wound healing, and well-being in EB patients and reduced concomitant medication use. Nevertheless, a direct relation between the use of CBMs and reduction of the above-mentioned symptoms cannot be proven by these data. Therefore, future controlled studies using pharmaceutically standardised CBM preparations in EB are warranted to delineate the risks and benefits of CBMs.

Keywords: Epidermolysis bullosa, Cannabinoid-based medicines, Symptom alleviation, Patient driven research, Genodermatoses, Survey, Pain, Itch, Wounds

*Correspondence: n.h.b.schrader@umcg.nl

${ }^{\dagger}$ Nicholas H. B. Schräder and Emily S. Gorell have contributed equally to the work

${ }^{1}$ Department of Dermatology, University Medical Centre Groningen, University of Groningen, Groningen, The Netherlands

Full list of author information is available at the end of the article

\section{Background}

Epidermolysis bullosa (EB) is a group of clinically and genetically heterogeneous genetic skin conditions whereby patients have fragile skin and mucosae that blister with slight trauma [1]. EB is characterized by repeated blistering and wounding, often with 
impaired wound healing, leading to a cascade of secondary problems, including chronic wounds, scarring, deformities, infections and extracutaneous symptoms [2]. EB is divided into types according to the level of blister formation, including EB simplex (EBS), junctional EB (JEB) and dystrophic EB (DEB), which is further subdivided into recessive DEB (RDEB) and dominant DEB (DDEB) [1].

Pain and pruritus have a significant impact on quality of life in EB patients; two of the top three unmet needs for EB patients include effective pain and pruritus treatments [3-6]. EB patients and clinicians continually seek out novel treatments to improve symptomatic care and quality of life. Two recent case series have brought to light the use of cannabinoidbased medicines (CBMs) in the EB-care setting [7-9]. However, the therapeutic potential and risks of such drugs have yet to be delineated in the context of EB care.

Cannabinoids, whether endogenous (endocannabinoids), plant-derived (phytocannabinoids), or synthetic, are molecules that activate various cascading pathways through endogenous cannabinoid-binding receptors (CB1 and CB2) and transient receptor potential (TRP) ion channels within the endocannabinoid system (ECS) [10]. These receptors are ubiquitous in the central and peripheral nervous systems, but are also localized to the immune system and skin [10-12]. Over 100 cannabinoids have been isolated, however $\triangle 9$-tetrahydrocannabinol (THC) and cannabidiol (CBD) are the most abundant and well-studied active cannabinoids [13]. THC, known for its psychoactive effects, is a partial agonist of CB1 and CB2. CBD, which is psychotropically inert, antagonizes THC effects, improving the tolerability of THC when co-administered [14].

Pain treatment with CBMs has been well-described. Literature reviews of clinical studies have shown CBMs to be tolerable, reasonable therapeutic options for chronic pain in various conditions [15-17]. On the contrary, the effects of CBMs on pruritus lack sufficient clinical studies, but underlying mechanisms are attributed to neuronal activation, and local effects on keratinocytes and mast cells [18-22]. The modulation of pain and pruritus through CB1/CB2 ligation warrants further research on their potential role in alleviating symptoms and has the potential to become a novel target for treatment in EB $[9,23]$.

To improve our understanding of CBM treatments currently being used in $\mathrm{EB}$, and give direction to future research, we report the results of a survey aimed at highlighting patient-reported CBM-use, and selfreported effects on their EB.

\section{Patients and methods}

Eligibility included all types of inherited EB, all ages, and any geographic location. The survey was available online in English. Caregivers or parents could complete the survey on a patient's behalf. Participants 18 years old or older provided electronic informed consent. Assent and parental informed consent were completed for respondents aged 7-18 years. Parental informed consent was obtained for participants less than 7 years old.

The survey link was shared among EB-related social media groups and EB non-profit organization web pages and newsletters. Survey information was distributed among professional networks (EB Clinical Research Consortium and EB Clinical Network) who shared the information with EB patients.

The cross-sectional online survey obtained selfreported data on demographics, disease characteristics, CBM characteristics, effects of CBMs on EB symptoms, side effects, and changes in concomitant medication use (Additional file 1: Appendix 1). Survey questions were developed based on previously reported effects of CBMs on EB and other conditions, as well as input from EB patients and expert physicians [7, 8]. As this was an internationally disseminated survey, given the potential illegality of CBMs, it was designed to uphold participant anonymity. The survey utilized skip logic whereby pertinent follow up responses could be elicited. Retrospective pain and pruritus levels pre- and post-CBM use were collected as numerical rating scales (NRS), ranging from 0 to 10 [24]. The Wong-Baker FACES scale was included to assist with self-reporting of pain [25]. A 5-point Likert scale was used to assess improvement or worsening of EB symptoms; a 6-point Likert scale was used to assess medication changes. Options for "Not Applicable" were included for EB symptoms and medication use. An option to upload the CBM product label was included to confirm reported content of the product(s) used. Respondents had the option to include free text comments, which were reviewed by study staff. Qualitative answers were assessed for predominant themes.

Survey responses were collected, and data was managed using REDCap (Research Electronic Data Capture) secure electronic data capture tools hosted at Stanford University. Both the study and survey instrument were approved by the Stanford Institutional Review Board (Protocol \#53145) and the Groningen Institutional Ethics Review Board. Survey responses were collected from March through August 2020.

To ensure that only unique survey completions were included, surveys with identical responses for demographics or qualitative responses were identified and reviewed; the survey with the fewest completed questions was removed. Each individual survey was reviewed 
to confirm that responses appeared credible. Survey respondents that reported no previous or current CBM use were excluded. Incomplete surveys were included, however those that failed to complete at least the sections on CBM characteristics were excluded.

Levels of pain and pruritus pre- and post-CBM use were compared by utilizing non-parametric tests. Nonparametric testing was also applied to group comparisons. For costs, class midpoints from ordinal responses were extracted, and foreign currencies converted to United States Dollars based on exchange rates in December 2020. Statistical analyses were performed using SPSS (version 23.0).

\section{Results}

\section{Demographics}

In total, 155 surveys were returned. Of these, 84 surveys were excluded based on either incompletion $(n=42)$, no CBM use reported $(n=40)$, or were identified as duplicates $(n=2)$. The remaining $71(45.8 \%)$ survey responses comprised the analyzed study cohort (Table 1). Surveys were completed more frequently by EB patients (43/71, $60.6 \%$ ) than parents/caregivers (28/71, 39.4\%). Most responses came from North America (62/71, 87.3\%) but the cohort included inhabitants of five continents. The majority were $\geq 18$ years old $(45 / 71,63.4 \%)$. Participants with RDEB $(37 / 51,52.1 \%)$ comprised the largest represented EB type. Most participants reported moderate to very severe $\mathrm{EB}$ severity (58/71, 81.7\%). The vast majority of participants $(62 / 71,87.3 \%)$ currently used CBMs, versus those that had stopped using CBMs. Reasons for discontinuation included: CBMs were too expensive $(n=4)$, a better medication was found $(n=3)$, and that CBMs were illegal $(\mathrm{n}=1)$. The median monthly costs for CBMs were USD \$75.00 (IQR \$66.84-\$150).

\section{Cannabinoid-based medicine characteristics}

Participants reported characteristics of the CBMs administered and were able to report on multiple CBMs (Table 2). Oil/paste, flos and edible (infused or cooked into foods) were the most prevalent CBM preparations. On average, individual participants administered 2.4 ( \pm 1.5$)$ CBM preparation types. Topical (36/60, $60.0 \%)$ and ingested $(36 / 60,60.0 \%)$ were the most frequently encountered routes of administration. On average, participants used $2.1( \pm 1.1)$ administration routes. More respondents $(18 / 44,40.9 \%)$ preferred the inhaled route compared to other routes. For participants under 13 years, topical administration was most frequently used (12/19, 63.2\%).

Most commonly, CBM products contained both THC and CBD (41/118, 34.7\%), followed by CBD-only (24/118, $20.3 \%)$ and THC-only (18/118, 15.3\%). An unknown cannabinoid content was reported for $29.7 \%$ of CBMs (35/118). The largest group within respondents under 13 years used CBD-only (9/19, 47.4\%).

The majority of participants $(34 / 51,66.7 \%)$ administered CBMs at least once daily. The largest group reported that their dose of CBM fluctuated over time (17/52, 32.7\%), followed by both stable and increased (13/52, 25.0\%), and decreased (6/52, 11.5\%). For frequencies of CBM usage, most participants reported either stable or fluctuating (17/52, 32.7\%) frequencies.

CBMs were acquired primarily through dispensaries (31/50, 47.0\%) and social connections (19/50, 28.8\%). A minority of CBMs were prescribed $(12 / 50,24.0 \%)$, however most participants $(28 / 50,73.7 \%)$ reported that their clinicians were aware of their CBM use. Within the group of 12 participants using prescribed CBMs, 9 were from the United States, 2 from the Netherlands, and 1 from Australia.

Reasons for initiating CBM use were reported by 63 patients as a qualitative open-ended response. Of these responses, the most cited reason to start using a CBM was to treat pain $(n=40)$, and other EB symptoms, such as wound healing $(n=11)$, to improve sleep $(n=5)$, and to treat pruritus $(\mathrm{n}=5)$. Additionally, respondents reported starting CBM as an alternative to opiates $(n=8)$, inadequate control with non-CBM regimen $(n=7)$, and intolerable side effects for non-CBM regimen $(n=6)$.

\section{Reported effects of cannabinoid-based medicines}

Statistically significant reductions in self-reported pain and pruritus were reported retrospectively following CBM use (median pain change-score: -3 , IQR: -2 to -4 [ $\mathrm{p}<0.001,95 \%$ CI 2.95-4.04], median pruritus change-score: -3 , IQR: -1.25 to -5 [p $<0.001$, 95\% CI 2.59-3.10]) (Fig. 1). There were no significant differences in the change scores for pain and pruritus between $E B$ types (pain: $p=0.837$, pruritus: $p=0.864$ ) or self-reported disease severity (pain: $\mathrm{p}=0.644$, pruritus: $\mathrm{p}=0.962$ ). As most participants reported multiple CBM routes of administration and formulations, it was not possible to analyse degrees of improvement based on these variables.

The vast majority of participants reported improvement (much improved or a little improved) in overall symptoms (46/48, 95.8\%), overall pain $(45 / 48,93.8 \%)$ and overall pruritus $(40 / 44,90.9 \%)$ from CBM administration (Fig. 2). To a lesser extent, skin inflammation (34/47, $72.3 \%)$ and wound healing time $(29 / 48,60.4 \%)$ were reportedly improved with CBMs.

Within effects specific to pain types and qualities, most participants reported improvements in background pain (39/41, 95.1\%), pain related to movement (38/42, 90.5\%), pain located at wounds (39/46, 84.8\%), 
Table 1 Demographics and characteristics of survey respondents

\begin{tabular}{|c|c|c|c|c|c|}
\hline & Total cohort & RDEB & DDEB & EBS & JEB \\
\hline $\begin{array}{l}\text { Participants enrolled, } \\
\text { n (\%) }\end{array}$ & $71(100.0)$ & $37(52.1)$ & $8(11.3)$ & $17(23.9)$ & $8(11.3)$ \\
\hline \multicolumn{6}{|l|}{ Participant role, n (\%) } \\
\hline Patient & $43(60.6)$ & $18(48.6)$ & $6(75.0)$ & $13(76.5)$ & $5(62.5)$ \\
\hline $\begin{array}{l}\text { Parent/guardian/car- } \\
\text { egiver }\end{array}$ & $28(39.4)$ & $19(51.4)$ & $2(25.0)$ & $4(23.5)$ & $3(37.5)$ \\
\hline \multicolumn{6}{|l|}{ Age, $n(\%)$} \\
\hline$<7$ & $11(15.5)$ & $5(13.5)$ & $1(12.5)$ & $3(17.6)$ & $2(25.0)$ \\
\hline $7-12$ & $8(11.3)$ & $7(18.9)$ & - & $1(5.9)$ & - \\
\hline $13-17$ & $7(9.9)$ & $7(18.9)$ & - & - & - \\
\hline $18-25$ & $10(14.1)$ & $7(18.9)$ & $3(37.5)$ & - & - \\
\hline $26-34$ & $14(19.7)$ & $6(16.2)$ & $1(12.5)$ & $5(29.4)$ & $2(25.0)$ \\
\hline$>34$ & $21(29.6)$ & $5(13.5)$ & $3(37.5)$ & $8(47.1)$ & $4(50.0)$ \\
\hline \multicolumn{6}{|l|}{ Sex, n (\%) } \\
\hline Male & $40(56.3)$ & $25(67.6)$ & $4(50.0)$ & $7(41.2)$ & $3(37.5)$ \\
\hline Female & $31(43.7)$ & $12(32.4)$ & $4(50.0)$ & $10(58.8)$ & $5(62.5)$ \\
\hline \multicolumn{6}{|c|}{ Geographic region, $\mathrm{n}(\%)$} \\
\hline North America & $62(87.3)$ & $33(89.2)$ & $7(87.5)$ & $16(94.1)$ & $5(62.5)$ \\
\hline Europe & $5(7.0)$ & $2(5.4)$ & $1(12.5)$ & $1(5.9)$ & $1(12.5)$ \\
\hline Oceania & $2(2.8)$ & $1(2.7)$ & - & - & $1(12.5)$ \\
\hline Asia & $1(1.4)$ & $1(2.7)$ & - & - & - \\
\hline Africa & $1(1.4)$ & - & - & - & $1(12.5)$ \\
\hline \multicolumn{6}{|c|}{ Self-reported severity, $\mathrm{n}(\%)$} \\
\hline Very mild & $1(1.4)$ & - & - & $1(5.9)$ & - \\
\hline Mild & $12(16.9)$ & $2(5.4)$ & $3(37.5)$ & $7(41.2)$ & - \\
\hline Moderate & $22(31.0)$ & $9(24.3)$ & $3(37.5)$ & $6(35.3)$ & $3(37.5)$ \\
\hline Severe & $24(33.8)$ & $18(48.6)$ & $1(12.5)$ & $3(17.6)$ & $2(25.0)$ \\
\hline Very severe & $12(16.9)$ & $8(21.6)$ & $1(12.5)$ & - & $3(37.5)$ \\
\hline \multicolumn{6}{|l|}{ CBM-use, n (\%) } \\
\hline $\begin{array}{l}\text { Currently administering } \\
\text { CBM }\end{array}$ & $62(87.3)$ & $33(89.2)$ & $6(75.0)$ & $14(82.4)$ & $8(100.0)$ \\
\hline $\begin{array}{l}\text { Previously administered } \\
\text { CBM }\end{array}$ & $9(12.7)$ & $4(10.8)$ & $2(25.0)$ & $3(17.6)$ & - \\
\hline $\begin{array}{l}\text { Age when CBM } \\
\text { started, median (IQR) }\end{array}$ & $19(14.0-26.0)$ & $16(11.8-24.3)$ & $18(10.0-21.5)$ & $25(12.5-38.0)$ & $25.5(21.8-44.5)$ \\
\hline $\begin{array}{l}\text { Monthly costs in USD, } \\
\text { median (IQR) }(n=49)\end{array}$ & $\$ 75.00(\$ 66.84-\$ 150)$ & $\begin{array}{l}\$ 117.33(\$ 75.00- \\
\$ 150.00)\end{array}$ & $\begin{array}{l}\$ 112.50(\$ 33.42- \\
\$ 150.00)\end{array}$ & $\$ 75.00(\$ 27.15-\$ 150.00)$ & $\$ 75.00(\$ 25.00-\$ 91.93)$ \\
\hline
\end{tabular}

Demographics, participant reported EB characteristics and CBM-use status of survey respondents $(n=71)$, grouped by EB types. One participant with unknown EB type was removed from EB type columns

RDEB: recessive dystrophic epidermolysis bullosa, DDEB: dominant dystrophic epidermolysis bullosa, EBS: epidermolysis bullosa simplex, JEB: junctional epidermolysis bullosa, CBM: cannabinoid-based medicine, IQR: inter-quartile range, USD: United States dollar

pain during dressing changes $(35 / 42,83.3 \%)$ as well as itchy (41/43, 95.3\%), shooting (31/36, 86.1\%), stabbing $(31 / 35,88.6 \%)$ and burning $(30 / 36,83.3 \%)$ qualities of pain.

The ability to relax (41/43, 95.3\%), overall mood (36/39, 92.3\%), improvement in anxiety (32/36, 88.9\%), sleep (39/43, 90.7\%), and ability to move around (37/43, $86 \%)$ were the most frequently improved effects related to participants' well-being.
Of participants who reported having skin cancer $(n=10)$, two indicated reduced cancer growth. Of the seven who reported experiencing pain from skin cancer, six $(85.7 \%)$ denoted decreased pain from skin cancer with CBM use.

Notably, a small fraction of responses indicated worsening in overall symptoms $(1 / 48,2 \%)$, overall pruritus $(1 / 44,2.3 \%)$, wound infections $(1 / 44,2.3 \%)$, pain during dressing changes $(1 / 42,2.4 \%)$, pain with an itchy quality 
Table 2 Characteristics of cannabinoid-based medicines

\begin{tabular}{|c|c|c|c|}
\hline $\begin{array}{l}\text { Survey item subject (number of responses to } \\
\text { item) }\end{array}$ & Multiple choice options for survey responses & All respondents $(\%)$ & $\begin{array}{l}\text { Respondents }<13 \text { years } \\
(\%)\end{array}$ \\
\hline \multirow{8}{*}{$\begin{array}{l}\text { CBM type* } \\
N \text { all respondents }=60 \\
N<13 y=19\end{array}$} & Oil/paste & $46(76.7)$ & $11(57.9)$ \\
\hline & Dried flower/Flos & $33(55.0)$ & $2(10.5)$ \\
\hline & Edible & $29(48.3)$ & $2(10.5)$ \\
\hline & Tincture & $17(28.3)$ & $3(15.8)$ \\
\hline & Other liquid form & $11(18.3)$ & $1(5.3)$ \\
\hline & Pill & $5(8.3)$ & - \\
\hline & Total CBM types used & 141 & 19 \\
\hline & Mean per participant & $2.4( \pm 1.5)$ & $1.0( \pm 0.7)$ \\
\hline \multirow{6}{*}{$\begin{array}{l}\text { CBM route of administration* } \\
N \text { all respondents }=60 \\
N<13 y=19\end{array}$} & Topical & $36(60.0)$ & $12(63.2)$ \\
\hline & Ingested & $36(60.0)$ & $4(21.1)$ \\
\hline & Inhaled & $33(55.0)$ & - \\
\hline & Sublingual & $23(38.3)$ & $4(21.1)$ \\
\hline & Total routes reported & 128 & 20 \\
\hline & Mean per participant & $2.1( \pm 1.1)$ & $1.1( \pm 0.8)$ \\
\hline \multirow{7}{*}{$\begin{array}{l}\text { Topical CBM type* } \\
N \text { all respondents }=36 \\
N<13 y=19\end{array}$} & Cream & $24(66.7)$ & $8(42.1)$ \\
\hline & Oil & $23(63.9)$ & $6(31.6)$ \\
\hline & Lotion & $12(33.3)$ & $4(21.1)$ \\
\hline & Spray & $3(8.3)$ & $1(5.3)$ \\
\hline & Foam & - & - \\
\hline & Total types of topicals & 62 & 19 \\
\hline & Mean per participant & $1.7( \pm 1.1)$ & $1.1( \pm 1.2)$ \\
\hline \multirow{5}{*}{$\begin{array}{l}\text { Composition of CBM (THC vs. CBD)* } \\
\text { N All Respondents }=58 \\
N<13 y=19\end{array}$} & THC only & $18(15.3)$ & - \\
\hline & THC/CBD combination & $41(34.7)$ & $6(31.6)$ \\
\hline & CBD only & $24(20.3)$ & $9(47.4)$ \\
\hline & Unknown & $35(29.7)$ & $4(21.1)$ \\
\hline & Total compositions reported & 118 & 19 \\
\hline \multirow{6}{*}{$\begin{array}{l}\text { Current frequency of CBM administration } \\
N \text { all respondents }=51 \\
N<13 y=10\end{array}$} & Less than once per week & $5(9.8)$ & $2(20.0)$ \\
\hline & Once per week & $2(3.9)$ & - \\
\hline & Several times per week & $8(15.7)$ & $1(10.0)$ \\
\hline & Once per day & $15(29.4)$ & $4(40.0)$ \\
\hline & Several times per day & $19(37.3)$ & $2(20.0)$ \\
\hline & Unsure & $2(3.9)$ & $1(10.0)$ \\
\hline \multirow{5}{*}{$\begin{array}{l}\text { Change over time: dose of CBM } \\
N \text { all respondents }=52 \\
N<13 y=11\end{array}$} & Dose decreased & $6(11.5)$ & $1(9.1)$ \\
\hline & Dose remained stable & $13(25.0)$ & $2(18.2)$ \\
\hline & Dose increased & $13(25.0)$ & $4(36.4)$ \\
\hline & Dose fluctuated & $17(32.7)$ & $2(18.2)$ \\
\hline & Unsure & $3(5.8)$ & $2(18.2)$ \\
\hline \multirow{5}{*}{$\begin{array}{l}\text { Change over time: frequency of CBM administra- } \\
\text { tion } \\
N \text { all respondents }=52 \\
N<13 y=11\end{array}$} & Administered less frequently & $7(13.5)$ & $1(9.1)$ \\
\hline & Frequency remained stable & $17(32.7)$ & $3(27.3)$ \\
\hline & Administered more frequently & $9(17.3)$ & $2(18.2)$ \\
\hline & Administration frequency fluctuated & $17(32.7)$ & $4(36.4)$ \\
\hline & Unsure & $2(3.8)$ & 1 \\
\hline \multirow{7}{*}{$\begin{array}{l}\text { CBM source of acquisition* } \\
N \text { All Respondents }=50 \\
N<13 y=10\end{array}$} & Cannabis dispensary & $31(62.0)$ & $6(60.0)$ \\
\hline & Social connection & $19(38.0)$ & $2(20.0)$ \\
\hline & Internet web-shops & $6(12.0)$ & $4(40.0)$ \\
\hline & Medical pharmacy & $5(10.0)$ & $1(10.0)$ \\
\hline & Cultivated at home & $4(8.0)$ & - \\
\hline & Prefer not to answer & $1(2.0)$ & - \\
\hline & Total number of sources & $66(100)$ & $13(100)$ \\
\hline
\end{tabular}


Table 2 (continued)

\begin{tabular}{|c|c|c|c|}
\hline $\begin{array}{l}\text { Survey item subject (number of responses to } \\
\text { item) }\end{array}$ & Multiple choice options for survey responses & All respondents (\%) & $\begin{array}{l}\text { Respondents }<13 \text { years } \\
(\%)\end{array}$ \\
\hline \multirow{4}{*}{$\begin{array}{l}\text { Preferred route of administration } \\
N \text { all respondents }=44 \\
N<13 y=9\end{array}$} & Topical & $11(25.0)$ & \multirow[t]{2}{*}{$4(44.4)$} \\
\hline & Inhaled & $18(40.9)$ & \\
\hline & Ingested & $10(22.7)$ & $2(22.2)$ \\
\hline & Sublingual & $5(11.4)$ & $3(33.3)$ \\
\hline $\begin{array}{l}\text { Duration of use } \\
N \text { all respondents }=52 \\
N<13 y=11\end{array}$ & $\begin{array}{l}<6 \text { months } \\
6 \text { months }-1 \text { year } \\
1-5 \text { years } \\
>5 \text { years }\end{array}$ & $\begin{array}{l}13(25.0) \\
7(13.5) \\
18(34.6) \\
14(26.9)\end{array}$ & $\begin{array}{l}5(45.5) \\
4(36.4) \\
2(18.2) \\
-\end{array}$ \\
\hline \multirow{8}{*}{$\begin{array}{l}\text { Reported side effects* } \\
N \text { all respondents }=45 \\
N<13 y=9\end{array}$} & Dry mouth & $20(44.4)$ & - \\
\hline & Cough/wheezing & $13(28.8)$ & - \\
\hline & Dry/red eyes & $12(26.7)$ & $1(11.1)$ \\
\hline & Fatigue & $10(22.2)$ & - \\
\hline & Dizziness & $7(15.6)$ & - \\
\hline & Paranoia & $3(6.7)$ & - \\
\hline & Problems with memory/attention & $3(6.7)$ & - \\
\hline & Problems with coordination & $3(6.7)$ & - \\
\hline \multirow{2}{*}{$\begin{array}{l}\text { CBM prescribed by physician } \\
N \text { all respondents }=50 \\
N<13 y=10\end{array}$} & Yes & $12(24.0)$ & $1(10.0)$ \\
\hline & No & $38(76.0)$ & $9(90.0)$ \\
\hline \multirow{2}{*}{$\begin{array}{l}\text { Physician aware of CBM use } \\
N \text { all respondents }=50 \\
N<13 y=9\end{array}$} & Yes & $28(73.7)$ & $7(77.8)$ \\
\hline & No & $10(26.3)$ & $2(22.2)$ \\
\hline
\end{tabular}

Reported cannabinoid-based medicines (CBMs) and characteristics of administration in the total cohort. * Indicates that participants were able to select more than one response. Participants were not required to respond to each item. Administration by way of suppositories were not reported by any participant

THC: delta-9-tetrahydrocannabinol, CBD: cannabidiol

$(1 / 43,2.3 \%)$, pain in the mouth/throat $(1 / 31,3.2 \%)$, appetite (1/40, 2.5\%), and energy levels (5/41, 12.2\%) (Fig. 2).

Effects of CBM administration on pain and pruritus treatments were highlighted by cessation of, or reduction in the use of, opioids $(12 / 15,80.0 \%)$, over the counter pain medications $(18 / 23,78.3 \%)$ and anti-itch medications $(17 / 25,68.0 \%$, Fig. 3). Of participants who required wheelchair assistance, most $(14 / 25,56.0 \%)$ reported a reduced need to use a wheelchair.

The most commonly encountered side-effect from CBM administration was $d r y$ mouth reported by $44 \%$ of participants (Table 2). Cough/wheezing (29\%), and dry/ red eyes (27\%) were the next most prevalent side effects.

\section{Discussion}

We report the first international summary of patient reported $\mathrm{CBM}$ use in $\mathrm{EB}$. The literature assessing CBM treatments in EB is non-existent. To date only two small ( $n=6$ total) retrospective case-series have been published, which highlighted various cannabinoid compositions of CBMs, administered topically or sublingually $[7,8]$. The reported effects here were strikingly similar, characterized by reductions in pain, pruritus, and in the use of other systemic medications, such as opioids. In EB, pain treatment guidelines have noted the use of CBMs as potential modalities, but evidence remains empirical [4].

EB is an immensely burdensome condition from birth, which, in the absence of a cure or highly effective treatments, means that patients are on a continuous journey to find relief in novel, repurposed or alternative treatments [26]. It is not surprising that EB patients willingly experiment with treatments that may not have been thoroughly investigated, such as CBMs that contain CBD and THC. Several participants reportedly sought out CBMs as an alternative to conventional medications (such as opioids), or other substances (such as alcohol). Reassuringly the majority informed their clinicians of their CBM use. However, even with promising anecdotes, before any clinical decisions can be made regarding their safety, tolerability and effectiveness, CBM treatment risks and benefits should be clearly delineated.

Most of the responses in this study were from North America, despite international outreach to recruit respondents; four other continents were also represented. Due to the extensive availability of CBMs in the United States and Canada, it was fitting that the majority of responses originated from these two countries [27, 28]. 


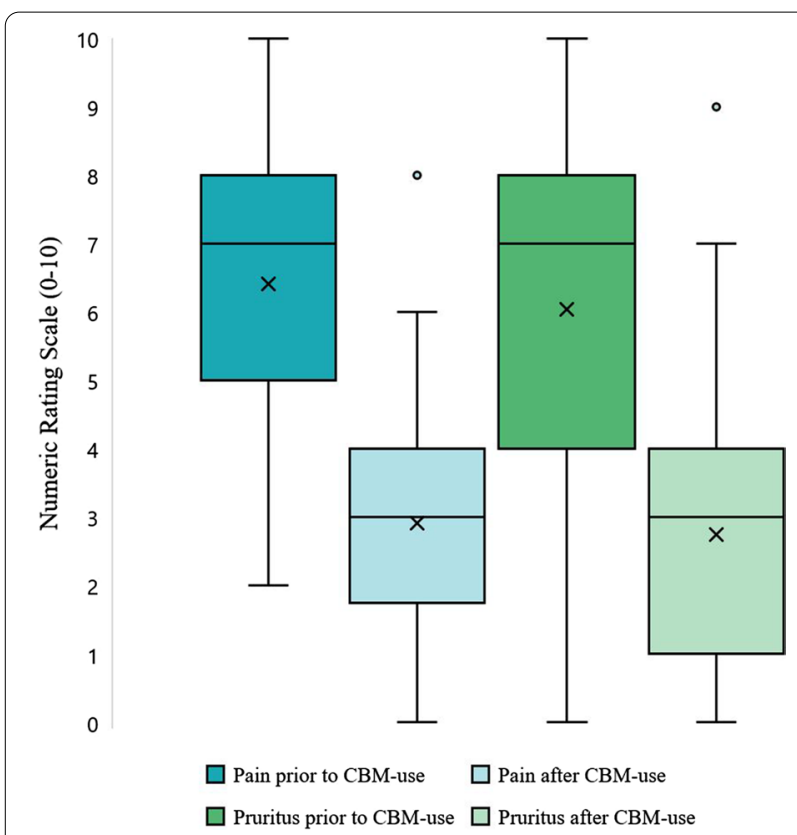

Fig. 1 Boxplot of numeric rating scale (NRS 0-10) scores for pain and pruritus prior to- and after cannabinoid-based medication (CBM) administration. Note the improvement in the perception of both pain and pruritus following CBM use. Significant differences $(p<0.001)$ were seen for changes in pain and pruritus (prior to CBM-use vs. after (BM-use)

The vast majority of respondents had RDEB, and half of participants reported their EB as severe or very severe. Although each EB type has a unique pathophysiology, some symptoms, such as blistering and wound formation, overlap. Patients with any EB type can perceive their disease as severe, influenced by environmental and psychosocial factors; it appears that this perception of disease severity likely contributed to participant willingness to try CBMs [29]. However, reported improvement in symptomatology was not exclusive to RDEB, nor was it seen only in patients who self-classified as severe.

The most commonly cited reason for discontinuing CBMs was price, with an average of USD \$75 spent monthly. A downside to the burgeoning market of CBM products is the expense, especially in a population already significantly impacted by the financial burden of caring for their disease [30, 31]. Notably, some participants in the United States reported that they started using CBMs due to difficulties in obtaining conventional allopathic medications, either due to price, lack of insurance coverage, or difficulty obtaining prescriptions (often narcotics).

\section{Characteristics of cannabinoid-based medicines}

The administration routes reported in this study were predominantly inhaled, topical and oral, closely followed by sublingual. Generally, inhaled and sublingual CBM administration lead to the fastest plasma cannabinoid peaks, due to the direct absorption of cannabinoids into the vasculature, allowing for efficient dose-titration in shorter time-frames [32, 33]. This may partially explain why participants in this study mostly preferred the inhaled route.

Topical CBMs, however, have local effects, whereby psychotropic or systemic side-effects are very limited, but the mechanisms of peripheral CB1/2 binding may differ from systemic CBMs [34]. Patients or caregivers may initiate these CBM treatments due to the ease of topical administration, the high burden of skin related problems in EB, and the desire to minimize side effects, as was reported in a case study [7]. We found that topical administration of CBD-only products were the predominant CBMs used for children with EB. The increased availability of legal CBD-only products may have also contributed to the increased use of these products compared to other CBMs.

Most participants administered CBMs at least daily, underscoring the degree of intervention required to manage EB symptoms. The reported dosages and frequency of use showed heterogeneity in change over time. A small, yet significant number of participants noted an increase in both administered dose (13/52, 25\%) and administration frequency $(9 / 52,17.3 \%)$ over time. Typically, tolerance to the efficacy of CBMs containing THC and CBD, has not been observed [35-38]. However, tolerance is developed to known effects from cannabinoid receptor agonism such as impaired neurocognition and cardiovascular changes [39]. The changes in dose and frequency of administration seen in this study may also be due to the dynamic natural history of symptoms in EB, such as the development or resolution of blistering and chronic or recurrent wounds, fluctuations in pain and pruritus, or disease progression, amongst an array of factors [2, 40].

There is ongoing debate as to the synergistic value of combining multiple cannabinoids (i.e., both CBD and THC) in CBM treatments [41]. The phytocannabinoids THC and CBD, amongst other cannabinoids, have unique binding properties with CB1/2 and other ECSassociated receptors. THC is proposed as the molecule central to CBM pain treatments, however current recommendations include adding CBD to mitigate THCmediated side effects [42]. Increasing ratios of THC:CBD linearly improve patient-reported effects for numerous indications, but could reduce these effects at too high a ratio [43]. The varying cannabinoid compositions used by participants in our study may be due to differences in legality, availability, recommendations from peers, or settings where participants acquired CBMs. Standardizing CBM compositions, as well as finding the optimal ratios 


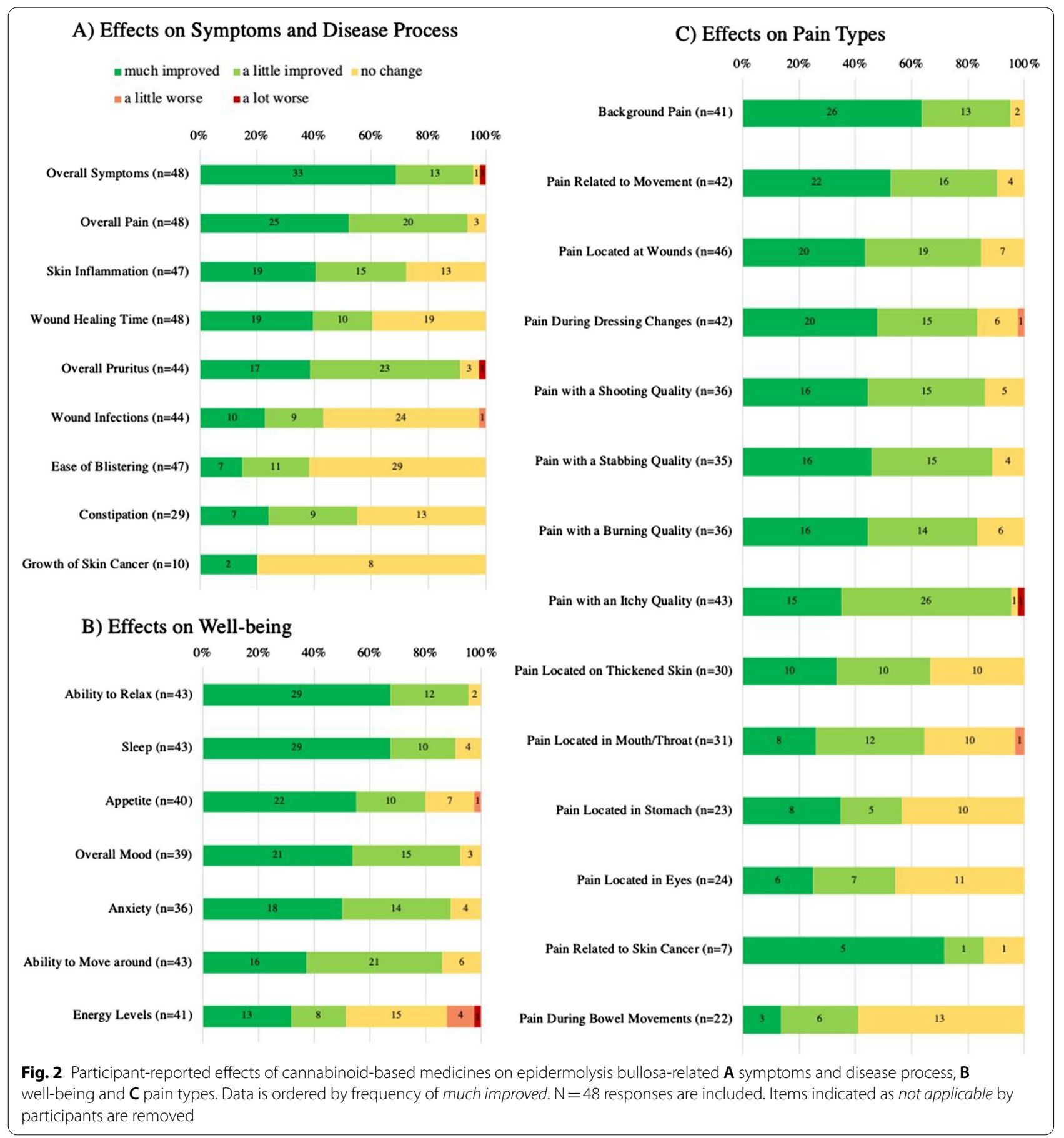

for such treatments will help gain more accurate insights into CBM effects in EB.

\section{Effects on symptoms of epidermolysis bullosa}

Both the highest prevalence and proportion of reported improvement from CBMs was in overall symptoms.
This was closely followed by overall pain and pruritus. As participants indicated multidimensional positive effects of CBMs in this study, it is likely that the synergistic improvements in multiple aspects of their disease may together be more clinically meaningful than each individual effect. Contrastingly, very few patients reported worsening of symptoms. 


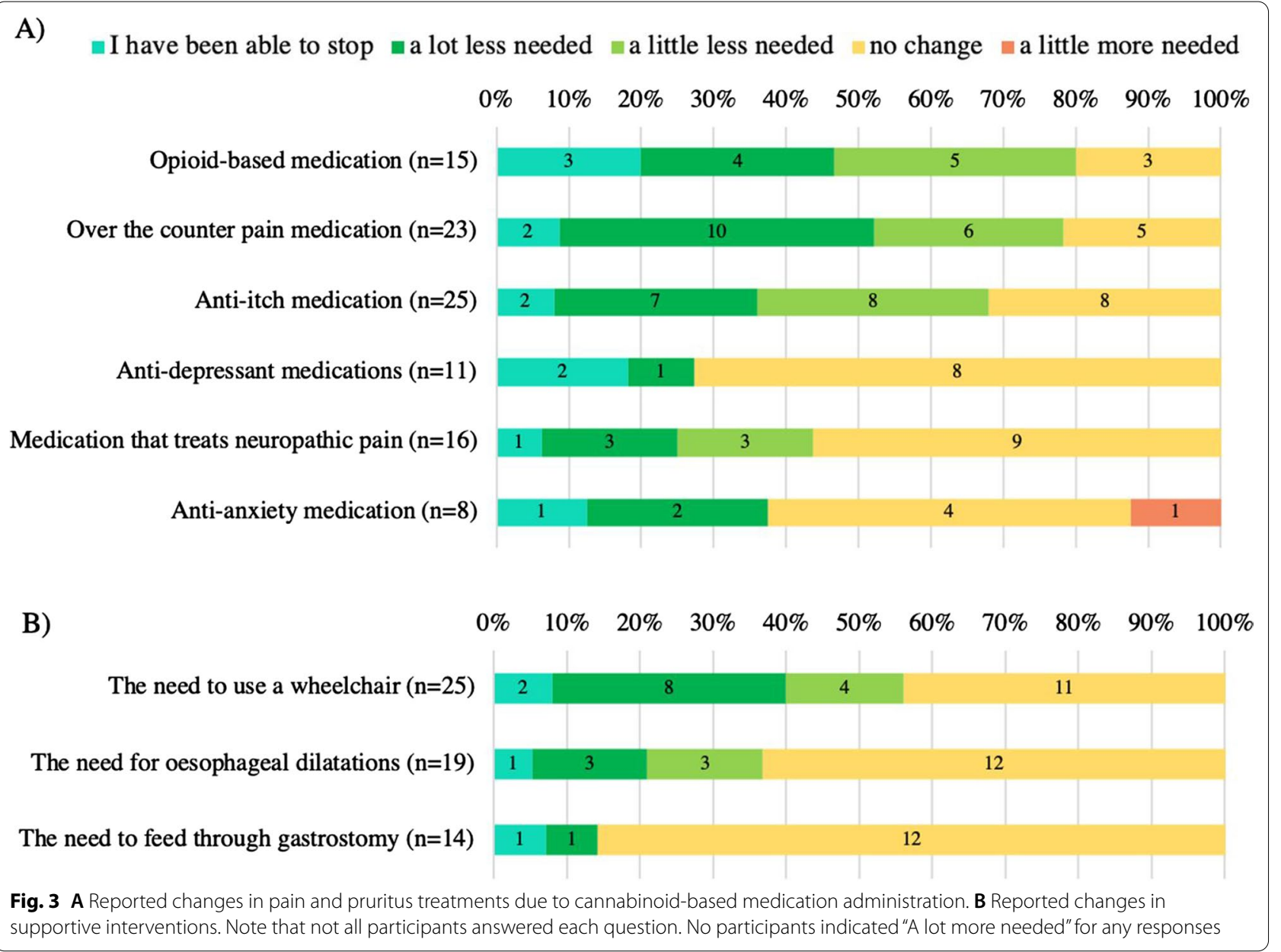

The greatest degrees of improvement were observed in background pain, pain related to movement and wound pain. Some of the most well studied indications for CBM interventions are chronic pain conditions $[17,44]$. The consistently superior results of CBMs, containing both $\mathrm{THC}$ and $\mathrm{CBD}$, versus placebo in non-EB neuropathic pain studies is promising, but is overshadowed by lack of high-quality evidence [45]. To date, CBMs have, at most, moderate quality evidence supporting their effectiveness for chronic pain. Pain, however, is a complex experience, influenced by neurobiological and psychosocial mechanisms [46]. The aetiology of pain in EB can be multifactorial including nociceptive pain (e.g., acute wounds, chronic wounds, dressing changes, surgical interventions, and extra-cutaneous sites of tissue damage), continuous background pain, neuropathic and nociplastic pain [4]. Due to the heterogeneity of pain mechanisms and responses to CBMs, establishing therapeutic mechanisms of CBMs in EB will be a challenging feat, but one deserving of additional study.
Many respondents reported overall reductions in pruritus. Patient-centred research has shown that pruritus is one of the most bothersome symptoms in EB [6, 47, 48]. Despite numerous treatment modalities, alleviation is challenging $[5,49]$. Thus, pruritus relief by way of intervention with CBMs could be especially meaningful to patients. Decreased pruritus was also noted in the previous case report of sublingual CBM use in EB patients [8]. However, one patient in our study did note increased pruritus as a side effect of CBMs. CBMs have reportedly decreased pruritus in multiple conditions such as atopic dermatitis, psoriasis, prurigo nodularis, uremic pruritus, and lichen amyloidosis [20,50]. It is postulated that the anti-pruritic actions of CBMs, like in pain, are due to effects within the ECS including ligation of CB1/2 receptors, and TRP channel modulation [19, 20, 22]. Additional studies are warranted to investigate pruritus alterations with $\mathrm{CBMs}$ in $\mathrm{EB}$, including elucidating the most effective routes of administration, and CBM compositions. 
The wound environment in EB consists of an interplay between intrinsically impaired wound healing, bacterial colonization, inflammation and the external wound environment [51]. The majority of participants reported improved wound healing and inflammation from CBMs. After tissue injury, the ECS plays an intricate role in the regulation of cytokines, nitrergic signalling and keratinocyte differentiation, through direct and indirect activation of cannabinoid receptor ligands [22, 52-54]. The two recent case series, coupled with our results deem further investigation necessary to address whether CBM modulation of the ECS is beneficial for EB wounds $[7,8]$. We do note that subjectively reported improvements in wound healing and inflammation could also be multifactorial, secondary to reductions in pain and/or pruritus. The natural history of EB wounds is often unpredictable and influenced by both biological and environmental factors [40, 55]. Future studies assessing the effects of CBMs on EB wounds should incorporate objective wound assessments.

The highest proportions of reductions in concomitant medications were seen in opioid-based analgesics, over the counter analgesics, and anti-itch medications. These treatments comprise one aspect of the multidimensional approach to symptomatic care in EB $[4,5]$. Yet, often in EB, conventional medications do not provide adequate effectiveness and are eclipsed by their burdensome short and long term side-effect profiles $[4,56]$. Identifying effective pain and pruritus treatments remains a research priority in EB $[29,57-59]$. Recent reviews suggest a prominent opioid-sparing effect from CBMs, warranting future clinical studies to investigate a causal relationship [60]. Although CBMs do not appear to alter the human pharmacokinetics of opioids, the ECS and endogenous opioid systems share neuroanatomical, neurochemical and pharmacological characteristics [61, 62]. The reduction of concomitant analgesic and anti-pruritic medication in this study seems promising, and mirror those effects previously reported wherein some patients were able to discontinue opioids $[7,8]$. However, it is of significant importance to obtain a clear picture of this phenomenon in EB by clarifying both the short and long-term risks and benefits of these medication regimen alterations.

Notably, of the respondents who required a wheelchair, more than half were able to decrease or even stop using their wheelchair. These respondents reported both improved energy levels and ability to move around. It is likely that these improvement are related to the reduction in symptomatic burdens, yet remains an important finding as increased mobility in EB has myriad conceivable benefits [63-65].
Ten participants reported skin cancer, of which two noted a reduction in growth with CBMs. There have been reports of anti-neoplastic effects of CBMs [12, $19,66]$. Unfortunately, we are not able to validate these findings, nor can we describe potential anti- or proneoplastic effects of CBMs, as no conclusive evidence has delineated the mechanisms of action, risks and benefits of such therapies in EB. Additionally, 6/7 participants reported reduced pain from skin cancer (the type of cancer was not specified), due to CBMs. Current literature suggests a role for CBMs in alleviating cancer-related pain in non-EB patients, however high quality evidence is lacking [67].

A large proportion of participants indicated a positive impact of CBMs on their well-being, of which the greatest reported improvements were for the ability to relax and sleep. Daily well-being has been given substantial consideration in EB best practice guidelines, and highlight the multidimensional burdens of living with EB [4, 68]. Whether these reported improvements are direct physiological effects of CBMs, or indirect through symptom alleviation, are not known and will require further investigation.

\section{Side effects}

In this study, dry mouth was the most commonly reported side-effect, likely due to inhibition of salivation through CB1/2 modulation in the salivary glands, via THC and CBD-induced agonism [69]. Dry eyes were also reported, probably through similar mechanisms in lacrimal glands. Ocular, oral, and dental sequelae of EB can be exacerbated by these effects, and thus should be considered prior to CBM administration [70]. However, overall, scientific literature points out that CBMs are generally well tolerated, as was reported by participants in this study [71].

An additional factor to consider, with specific regard to THC, is CBM dependence and withdrawal symptoms after therapy cessation, or periods of cessation, which were not incorporated in this study. The lifetime risk for dependence after cannabis use in recreational settings (high THC concentrations and aim to achieve psychotropic effects) is $8.9 \%$ [72]. The dependence risk whilst using CBMs for therapeutic goals is however unknown. Withdrawal symptoms may include irritability, anger, anxiety, sleep difficulty, decreased appetite and weight loss, and is dose-dependent [73]. Future prospective controlled studies taking withdrawal into account, may add value to the sparse evidence on withdrawal after clinically supervised CBM-use. 


\section{Limitations}

Given the potential illegality of CBM use, we implemented an anonymous online survey. Thus, we were unable to gauge the prevalence of CBM use, nor a response rate. During the data cleaning phase, the research team cross-checked individual responses, but could not completely validate the content of submitted surveys.

The number of included patients was small for English speaking countries outside North America and may be due to differences in availability of CBMs. Unfortunately, we must also accept that CBM use, even with clinical supervision, carries a stigma whereby patients may refrain from disclosing their use.

The composition of the CBM products used by patients is also not entirely clear. While we attempted to ascertain the cannabinoid compositions of CBMs by allowing participants to upload product labels, not every participant uploaded their label. Additionally, the commercial market regarding CBMs remains unregulated whereby cannabinoid compositions are non-standardised and may be inaccurately labelled $[74,75]$. In order to avoid health risks of accidentally administering unwanted additives, it is important for patients to acquire CBMs from reputable sources. This also underscores the potential benefit of regulation which would come with legalization of CBM products.

Furthermore, we unfortunately were unable to determine how cannabinoid concentrations (CBD versus $\mathrm{THC}$ ) and route of administration contributed to the perceived efficacy of CBMs reported in this study, as most participants used multiple products and routes of administration. Additional areas of future exploration include those that were alluded to by free text comments. Various participants reported that certain formulations and routes may be more efficacious for different symptoms and scenarios: "We find that a combination of ingested and topical applications works best, as each product helps with a different aspect of EB. Ingested products help with internal inflammation and pain control. Topical products help with localized pain and wound healing." Certainly, additional controlled studies are warranted to explore the complex relationships between cannabinoid compositions, routes of CBM administration, EB (patho-) physiologies and symptoms.

Finally, we recognize that there may have been a selection bias as the responses may comprise patients reporting very positive or very negative experiences. The retrospective reporting methodology makes this study vulnerable to a recall bias, and is also cause to interpret the findings with caution. Furthermore, these experiences are not standardized and there is a possibility that participants are benefitting from a placebo effect, underscoring the need for future controlled studies.

\section{Conclusion}

In conclusion, this study highlights the use and perceived multidimensional beneficial effects of treatments with CBMs by EB patients on EB symptoms and disease process. Future prospective controlled clinical studies are warranted to elucidate the potential role of CBMs in EB treatment.

\begin{abstract}
Abbreviations
CB1: Cannabinoid receptor 1; CB2: Cannabinoid receptor 2; CBD: Cannabidiol; CBM: Cannabinoid-based medicine; Cl: Confidence interval; DDEB: Dominant dystrophic epidermolysis bullosa; DEB: Dystrophic epidermolysis bullosa; EB: Epidermolysis bullosa; EBS: Epidermolysis bullosa simplex; ECS: Endocannabinoid system; IQR: Interquartile range; JEB: Junctional epidermolysis bullosa; NRS: Numeric rating scale; RDEB: Recessive dystrophic epidermolysis bullosa; SCC: Squamous cell carcinoma; SD: Standard deviation; THC: Delta-9-tetrahydrocannabinol; TRP: Transient receptor potential.
\end{abstract}

\section{Supplementary Information}

The online version contains supplementary material available at https://doi. org/10.1186/s13023-021-02010-0.

Additional file 1: Appendix 1: CBM\&EB REDCap Survey.

\section{Acknowledgements}

The authors are indebted to the Epidermolysis Bullosa Research Partnership, Epidermolysis Bullosa Medical Research Foundation, Epidermolysis Bullosa Clinical Research Consortium, Dystrophic Epidermolysis Bullosa Research Association International, Dystrophic Epidermolysis Bullosa Research Association United States, Dystrophic Epidermolysis Bullosa Research Association Canada, and CLINET. We would also like to thank the EB patients and their caregivers who completed our survey.

\section{Authors' contributions}

NHBS: Conceptualization-Lead, Data curation-Lead, Formal analysis-Lead, Funding acquisition-Equal, Investigation-Lead, Methodology-Lead, Project administration-Lead, Resources-Lead, Software-Lead, Validation-Equal, Visualization-Lead, Writing-original draft-Lead, Writing-review \& editing-Lead. ESG: Conceptualization-Lead, Data curation-Lead, Formal analysis-Lead, Funding acquisition-Lead, Investigation-Lead, Methodology-Lead, Project administration-Lead, Resources-Lead, Software-Lead, Validation-Equal, Visualization-Lead, Writing-original draft-Lead, Writing-review \& editing-Lead. RES: Formal analysis-Lead, Methodology-Lead, Software-Lead, Validation-Lead, Writing-original draft-Equal, Writing-review \& editing-Equal. JCD: Conceptualization-Supporting, Data curation-Supporting, Resources-Supporting, Supervision-Supporting, Writing-review \& editing-Equal. NH: Formal analysis-Supporting, Funding acquisition-Supporting, Project administration-Supporting, Writing-review \& editing-Supporting. VAP: Data curation-Supporting, Formal analysis-Supporting, Writing-review \& editing-Supporting. JYT: Conceptualization-Equal, Methodology-Supporting, Project administration-Equal, Supervision-Lead, Writing-original draft-Equal, Writing-review \& editing-Equal. APW: Conceptualization-Equal, Methodology-Supporting, Project administration-Supporting, Supervision-Lead, Writing-original draft-Equal, Writing-review \& editingEqual. MCB: Conceptualization-Equal, Methodology-Equal, Resources-Equal, Supervision-Lead, Writing-original draft-Equal, Writing-review \& editing-Equal. All authors read and approved the final manuscript.

\section{Funding}

Funded in part by a grant from Epidermolysis Bullosa Research Partnership.

Availability of data and materials

The datasets used and/or analysed during the current study are available from the corresponding author on reasonable request. 


\section{Declarations}

\section{Ethical approval and consent to participate}

Both the study and survey instrument were approved by the Stanford Institutional Review Board (Protocol \#53145) and the Groningen Institutional Ethics Review Board. Electronic informed consent was required for participation.

\section{Consent for publication}

All authors (as noted on page 1, title page, of the manuscript) read and approved the final manuscript, and consent for manuscript publication.

\section{Competing interests}

None to declare.

\section{Author details}

${ }^{1}$ Department of Dermatology, University Medical Centre Groningen, University of Groningen, Groningen, The Netherlands. ${ }^{2}$ Department of Dermatology, University of Cincinnati College of Medicine, Cincinnati, OH, USA. ${ }^{3}$ Department of Dermatology, Stanford University School of Medicine, Redwood City, CA, USA. ${ }^{4}$ Department of Health Sciences, Community and Occupational Medicine, University of Groningen, University Medical Centre Groningen, Groningen, The Netherlands. ${ }^{5}$ Columbia University School of Medicine, New York, NY, USA. ${ }^{6}$ Anaesthesiology Pain Centre, Department of Anaesthesiology, University Medical Centre Groningen, University of Groningen, Groningen, The Netherlands.

Received: 5 May 2021 Accepted: 24 August 2021

Published online: 06 September 2021

\section{References}

1. Has C, Bauer JW, Bodemer C, Bolling MC, Bruckner-Tuderman L, Diem A, et al. Consensus reclassification of inherited epidermolysis bullosa and other disorders with skin fragility. Br J Dermatol. 2020;183:614-27.

2. Boeira VLSY, Souza ES, Rocha BO, Oliveira PD, Oliveira MFSP, de Rêgo VRPA, et al. Inherited epidermolysis bullosa: clinical and therapeutic aspects. An Bras Dermatol. 2013;88:185-98.

3. Mauritz PJ, Jonkman MF, Visser SS, Finkenauer C, Duipmans JC, Hagedoorn M. Impact of painful wound care in epidermolysis bullosa during childhood: an interview study with adult patients and parents. Acta Derm Venereol. 2019;99:783-8.

4. Goldschneider KR, Good J, Harrop E, Liossi C, Lynch-Jordan A, Martinez $A E$, et al. Pain care for patients with epidermolysis bullosa: best care practice guidelines. BMC Med. 2014;12:178-201.

5. Papanikolaou M, Onoufriadis A, Mellerio JE, Nattkemper LA, Yosipovitch $G$, Steinhoff M, et al. Prevalence, pathophysiology and management of itch in epidermolysis bullosa. Br J Dermatol. 2021;184:816-25.

6. Danial C, Adeduntan R, Gorell ES, Lucky AW, Paller AS, Bruckner A, et al. Prevalence and characterization of pruritus in epidermolysis bullosa. Pediatr Dermatol. 2015;32:53-9.

7. Chelliah MP, Zinn Z, Khuu P, Teng JMC. Self-initiated use of topical cannabidiol oil for epidermolysis bullosa. Pediatr Dermatol. 2018;35:e224-7.

8. Schräder NHB, Duipmans JC, Molenbuur B, Wolff AP, Jonkman MF. Combined tetrahydrocannabinol and cannabidiol to treat pain in epidermolysis bullosa: a report of three cases. Br J Dermatol. 2019;180:922-4.

9. Martinez AE. Time to drop the stigma: cannabinoids are drugs that may alleviate pain in people with epidermolysis bullosa. Br J Dermatol. 2019;180:711-2.

10. Pacher P, Bátkai $S$, Kunos $G$. The endocannabinoid system as an emerging target of pharmacotherapy. Pharmacol Rev. 2006;58:389-462.

11. Bíró T, Tóth BI, Haskó G, Paus R, Pacher P. The endocannabinoid system of the skin in health and disease: novel perspectives and therapeutic opportunities. Trends Pharmacol Sci. 2009;30:411-20.

12. Tóth KF, Ádám D, Bíró T, Oláh A. Cannabinoid signaling in the skin: therapeutic potential of the "c(ut)annabinoid" system. Molecules. 2019;24:918

13. Andre CM, Hausman JF, Guerriero G. Cannabis sativa: the plant of the thousand and one molecules. Front Plant Sci. 2016;7:19.

14. MacCallum CA, Russo EB. Practical considerations in medical cannabis administration and dosing. Eur J Intern Med. 2018:49:12-9.
15. Lynch ME, Ware MA. Cannabinoids for the treatment of chronic noncancer pain: an updated systematic review of randomized controlled trials. J Neuroimmune Pharmacol. 2015;10:293-301.

16. Whiting PF, Wolff RF, Deshpande S, Di Nisio M, Duffy S, Hernandez AV, et al. Cannabinoids for medical use: a systematic review and meta-analysis. JAMA. 2015;313:2456-73.

17. Wong SSC, Chan WS, Cheung CW. Analgesic effects of cannabinoids for chronic non-cancer pain: a systematic review and meta-analysis with meta-regression. J Neuroimmune Pharmacol. 2020;15:801-29.

18. Liszewski W, Farah RS. Response to: "The role of cannabinoids in dermatology." J Am Acad Dermatol. 2017;77:e87-8.

19. Mounessa JS, Siegel JA, Dunnick CA, Dellavalle RP. The role of cannabinoids in dermatology. J Am Acad Dermatol. 2017;77:188-90.

20. Avila C, Massick S, Kaffenberger BH, Kwatra SG, Bechtel M. Cannabinoids for the treatment of chronic pruritus: a review. J Am Acad Dermatol. 2020;82:1205-12.

21. Jastrząb A, Gęgotek A, Skrzydlewska E. Cannabidiol regulates the expression of keratinocyte proteins involved in the inflammation process through transcriptional regulation. Cells. 2019;8:827.

22. Cintosun A, Lara-Corrales I, Pope E. Mechanisms of cannabinoids and potential applicability to skin diseases. Clin Drug Investig. 2020;40:293-304

23. Baswan SM, Klosner AE, Glynn K, Rajgopal A, Malik K, Yim S, et al. Therapeutic potential of cannabidiol (CBD) for skin health and disorders. Clin Cosmet Investig Dermatol. 2020;13:927-42.

24. Kimel M, Zeidler C, Kwon P, Revicki D, Ständer S. Validation of psychometric properties of the itch numeric rating scale for pruritus associated with prurigo nodularis: a secondary analysis of a randomized clinical trial. JAMA Dermatol. 2020;156:1354-8.

25. Garra G, Singer AJ, Domingo A, Thode HC. The wong-baker pain FACES scale measures pain, not fear. Pediatr Emerg Care. 2013;29:17-20.

26. Prodinger C, Diem A, Ude-Schoder K, Piñón-Hofbauer J, Kitzmueller S, Bauer JW, et al. Profiling trial burden and patients' attitudes to improve clinical research in epidermolysis bullosa. Orphanet J Rare Dis. 2020;15:182.

27. Bridgeman MB, Abazia DT. Medicinal cannabis: history, pharmacology, and implications for the acute care setting. PT. 2017:42:180-8.

28. Ko GD, Bober SL, Mindra S, Moreau JM. Medical cannabis - the Canadian perspective. J Pain Res. 2016;9:735-44

29. Bruckner AL, Losow M, Wisk J, Patel N, Reha A, Lagast H, et al. The challenges of living with and managing epidermolysis bullosa: Insights from patients and caregivers. Orphanet J Rare Dis. 2020;15:1

30. Gorell ES, Wolstencroft PW, de Souza MP, Murrell DF, Linos E, Tang JY. Financial burden of epidermolysis bullosa on patients in the United States. Pediatr Dermatol. 2020;37:1198-201.

31. Angelis A, Kanavos P, López-Bastida J, Linertová R, Oliva-Moreno J, Serrano-Aguilar P, et al. Social/economic costs and health-related quality of life in patients with epidermolysis bullosa in Europe. Eur J Heal Econ. 2016:17:31-42.

32. Karschner EL, Darwin WD, Goodwin RS, Wright S, Huestis MA. Plasma cannabinoid pharmacokinetics following controlled oral $\triangle 9$-tetrahydrocannabinol and oromucosal cannabis extract administration. Clin Chem. 2011:57:66-75.

33. Lucas CJ, Galettis P, Schneider J. The pharmacokinetics and the pharmacodynamics of cannabinoids. Br J Clin Pharmacol. 2018;84:2477-82.

34. Huestis MA. Human cannabinoid pharmacokinetics. Chem Biodivers. 2007:4:1770-804

35. De Hoop B, Heerdink ER, Hazekamp A. Medicinal cannabis on prescription in the Netherlands: statistics for 2003-2016. Cannabis Cannabinoid Res. 2018:3:54-5.

36. Ramaekers JG, Van Wel JH, Spronk DB, Toennes SW, Kuypers KPC, Theunissen EL, et al. Cannabis and tolerance: acute drug impairment as a function of cannabis use history. Sci Rep. 2016;6:26843.

37. Serpell MG, Notcutt W, Collin C. Sativex long-term use: an open-label trial in patients with spasticity due to multiple sclerosis. J Neurol. 2013:260:285-95.

38. Russo EB. Current therapeutic cannabis controversies and clinical trial design issues. Front Pharmacol. 2016;7:309.

39. Colizzi M, Bhattacharyya S. Cannabis use and the development of tolerance: a systematic review of human evidence. Neurosci Biobehav Rev. 2018:93:1-25. 
40. Solis DC, Teng C, Gorell ES, Barriga M, Nazaroff J, Li S, et al. Classification of two distinct wound types in recessive dystrophic epidermolysis bullosa: a retrospective and cohort natural history study. J Am Acad Dermatol [Internet]. 2020;Online ahead of print. https://www.sciencedirect.com/ science/article/pii/S0190962220325792

41. Russo EB. The case for the entourage effect and conventional breeding of clinical cannabis: no "Strain", no gain. Front Plant Sci. 2019;9:1969.

42. Fischer B, Russell C, Sabioni P, Van Den Brink W, Le Foll B, Hall W, et al. Lower-risk cannabis use guidelines: a comprehensive update of evidence and recommendations. Am J Public Health. 2017;107:e1-12.

43. Casarett DJ, Beliveau JN, Arbus MS. Benefit of tetrahydrocannabinol versus cannabidiol for common palliative care symptoms. J Palliat Med. 2019;22:1180-4

44. Banerjee S, McCormack S. Medical cannabis for the treatment of chronic pain: a review of clinical effectiveness and guidelines. CADTH (Canadian Agency Drugs Technol Heal Rapid Response Reports [Internet]. 2019;1-43. https://www.ncbi.nlm.nih.gov/books/NBK546424/

45. Mücke M, Phillips T, Radbruch L, Petzke F, Häuser W. Cannabis-based medicines for chronic neuropathic pain in adults. Cochrane Database Syst Rev. 2018;3:CD012182.

46. Dworkin RH, Bruehl S, Fillingim RB, Loeser JD, Terman GW, Turk DC. Multidimensional diagnostic criteria for chronic pain: introduction to the ACTTION-American Pain society pain taxonomy (AAPT). J Pain. 2016;17:T1-9.

47. Snauwaert JJLL, Yuen WY, Jonkman MF, Moons P, Naulaers G, Morren MA. Burden of itch in epidermolysis bullosa. Br J Dermatol. 2014;171:73-8.

48. Van Scheppingen C, Lettinga AT, Duipmans JC, Maathuis CGB, Jonkman MF. Main problems experienced by children with epidermolysis bullosa: a qualitative study with semi-structured interviews. Acta Derm Venereol. 2008:88:143-50

49. Danial C, Adeduntan R, Gorell ES, Lucky AW, Paller AS, Bruckner AL, et al. Evaluation of treatments for pruritus in epidermolysis bullosa. Pediatr Dermatol. 2015;32:628-34.

50. Roh YS, Sutaria N, Biles NF, Kwatra SG. Treatment of chronic pruritus with medical Marijuana. JAMA Dermatol. [Internet]. 2021;Online ahead of print. https://jamanetwork.com/journals/jamadermatology/fullarticle/ 2778442

51. El Hachem M, Zambruno G, Bourdon-Lanoy E, Ciasulli A, Buisson C, HadjRabia S, et al. Multicentre consensus recommendations for skin care in inherited epidermolysis bullosa. Orphanet J Rare Dis. 2014;9:76.

52. Maida V, Shi RB, Fazzari FGT, Zomparelli L. Topical cannabis-based medicines-a novel paradigm and treatment for non-uremic calciphylaxis leg ulcers: an open label trial. Int Wound J. 2020;17:1508-16.

53. Weigelt MA, Sivamani R, Lev-Tov H. The therapeutic potential of cannabinoids for integumentary wound management. Exp Dermatol. 2021;30:201-11.

54. Nagarkatti P, Pandey R, Rieder SA, Hegde VL, Nagarkatti M. Cannabinoids as novel anti-inflammatory drugs. Future Med Chem. 2009;1:1333-49. https://doi.org/10.4155/fmc.09.93.

55. Solis D, Nazaroff J, Dutt-Singkh Y, Choi S, Barriga M, Bailey-Healy I, et al 219 Natural history of chronic wounds in patients with recessive dystrophic epidermolysis bullosa. J Invest Dermatol. 2017;137:S37.

56. Goloff N, Dahl J. Successful opioid de-escalation with dexmedetomidine in a 1 year old with worsening pain and opioid-induced neurotoxicity. Pediatrics. 2018;142:meeting abstract 665.

57. Davila-Seijo P, Hernández-Martín A, Morcillo-Makow E, De LR, Domínguez $\mathrm{E}$, Romero $\mathrm{N}$, et al. Prioritization of therapy uncertainties in dystrophic epidermolysis bullosa: where should research direct to? An example of priority setting partnership in very rare disorders. Orphanet J Rare Dis. 2013;8:61.

58. Davila-Seijo P, Hernández-Martín Á, Morcillo-Makow E, Rajan C, GarcíaDoval I. Current dystrophic epidermolysis bullosa research does not match research needs perceived by patients and clinicians. J Am Acad Dermatol. 2014; 1008-11.

59. Brun J, Chiaverini C, Devos C, Leclerc-Mercier S, Mazereeuw J, Bourrat E, et al. Pain and quality of life evaluation in patients with localized epidermolysis bullosa simplex. Orphanet J Rare Dis. 2017;12:119.

60. Okusanya BO, Asaolu IO, Ehiri JE, Kimaru LJ, Okechukwu A, Rosales C. Medical cannabis for the reduction of opioid dosage in the treatment of non-cancer chronic pain: a systematic review. Syst Rev. 2020;9:1-8.

61. Befort K. Interactions of the opioid and cannabinoid systems in reward: Insights from knockout studies. Front Pharmacol. 2015;6:6.

62. Abrams DI, Couey P, Shade SB, Kelly ME, Benowitz NL. Cannabinoidopioid interaction in chronic pain. Clin Pharmacol Ther. 2011;90:844-51.

63. Chen JSC, Yang A, Murrell DF. Prevalence and pathogenesis of osteopenia and osteoporosis in epidermolysis bullosa: an evidence-based review. Exp Dermatol. 2019;28:1122-30

64. Hubbard LD, Mayre-Chilton K. Retrospective longitudinal study of osteoporosis in adults with recessive dystrophic epidermolysis bullosa. Clin Case Reports. 2019;7:58-63.

65. Martinez AE, Mellerio JE. Osteopenia and osteoporosis in epidermolysis bullosa. Dermatol Clin. 2010;28:353-5.

66. Milando R, Friedman A. Cannabinoids: potential role in inflammatory and neoplastic skin diseases. Am J Clin Dermatol. 2019;20:167-80.

67. Blake A, Wan BA, Malek L, DeAngelis C, Diaz P, Lao N, et al. A selective review of medical cannabis in cancer pain management. Ann Palliat Med. 2017;6:S215-22.

68. Martin K, Geuens S, Asche JK, Bodan R, Browne F, Downe A, et al. Psychosocial recommendations for the care of children and adults with epidermolysis bullosa and their family: evidence based guidelines. Orphanet J Rare Dis. 2019;14:133.

69. Prestifilippo JP, Fernández-Solari J, De La Cal C, Iribarne M, Suburo AM, Rettori $V$, et al. Inhibition of salivary secretion by activation of cannabinoid receptors. Exp Biol Med. 2006;231:1421-9.

70. Fine JD, Johnson LB, Weiner M, Stein A, Cash S, Deleoz J, et al. Eye involvement in inherited epidermolysis bullosa: experience of the national epidermolysis bullosa registry. Am J Ophthalmol. 2004;138:254-62.

71. Fitzcharles MA, Baerwald C, Ablin J, Häuser W. Efficacy, tolerability and safety of cannabinoids in chronic pain associated with rheumatic diseases (fibromyalgia syndrome, back pain, osteoarthritis, rheumatoid arthritis): a systematic review of randomized controlled trials. Schmerz. 2016;30:47-61.

72. Lopez-Quintero C, de Cobos JP, Hasin DS, Okuda M, Wang S, Grant BF, et al. Probability and predictors of transition from first use to dependence on nicotine, alcohol, cannabis, and cocaine: Results of the National Epidemiologic Survey on Alcohol and Related Conditions (NESARC). Drug Alcohol Depend. 2011;115:120-30.

73. Zehra A, Burns J, Liu CK, Manza P, Wiers CE, Volkow ND, et al. Cannabis addiction and the brain: a review. J Neuroimmune Pharmacol. 2018;13:438-52.

74. Hazekamp A. The trouble with CBD oil. Med Cannabis Cannabinoids. 2018;1:65-72.

75. Pavlovic R, Nenna G, Calvi L, Panseri S, Borgonovo G, Giupponi L, et al. Quality traits of "cannabidiol oils": Cannabinoids content, terpene fingerprint and oxidation stability of european commercially available preparations. Molecules. 2018;23:1230.

\section{Publisher's Note}

Springer Nature remains neutral with regard to jurisdictional claims in published maps and institutional affiliations. 